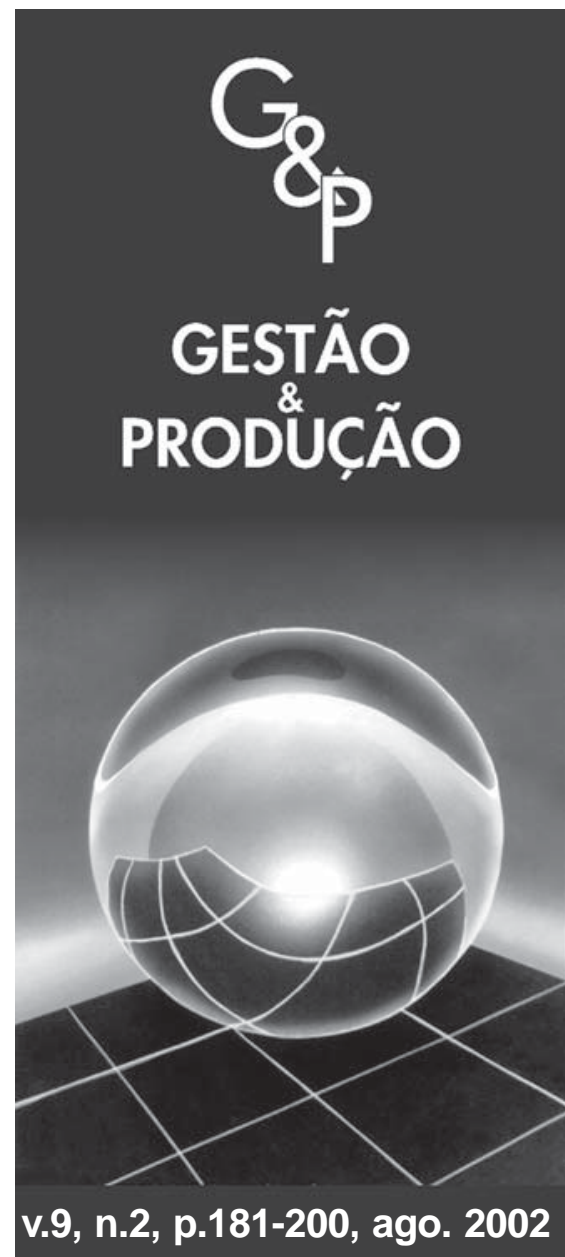

\title{
PROPOSTA DE UM MODELO CONCEITUAL PARA ANÁLISE DO SUCESSO DE PROJETOS DE TRANSFERÊNCIA DE TECNOLOGIA: ESTUDO EM EMPRESAS FARMACÊUTICAS
}

\author{
Vânia Passarini Takahashi \\ Faculdade de Ciências Farmacêuticas de \\ Ribeirão Preto, Universidade de São Paulo, \\ Av. Zeferino Vaz, s/no, Ribeirão Preto, SP, \\ CEP 14040-903, e-mail: vptakahs@usp.br \\ José Benedito Sacomano \\ Escola de Engenharia de São Carlos, \\ Universidade de São Paulo, \\ Engenharia de Produção, \\ Universidade Paulista, \\ e-mail: sacomano@zaz.com.br
}

\section{Resumo}

A aquisição de conhecimentos tecnológicos externos tem se tornado uma prática comum para as empresas farmacêuticas desenvolverem medicamentos mais eficazes e seguros. Visando colaborar para o maior conhecimento sobre o sucesso da transferência de tecnologia nessas empresas, foi revisado o tema fatores de sucesso na transferência de tecnologia e analisada a aplicabilidade de um modelo conceitual em três empresas no Canadá.

Palavras-chave: transferência de tecnologia, capacidades tecnológicas, capacidades gerenciais, empresas farmacêuticas.

\section{Introdução}

Numa indústria "baseada na ciência" como a farmacêutica a vantagem competitiva é conseguida por intermédio das inovações de seus produtos (Omta et al., 1994). Para sobreviver na indústria farmacêutica, as empresas têm de lançar um novo produto de cada três a cinco anos (Fitzgerald, 1992). Esse crescente esforço de inovação requer um grau de sofisticação tecnológico.

Segundo Tapon \& Thong (1999), está se tornando cada vez mais difícil para a empresa dominar sozinha a grande diversidade de 
conhecimento científico e tecnológico necessária para desenvolver novos medicamentos.

A aquisição de conhecimentos tecnológicos externos tem se tornado uma prática comum nas empresas farmacêuticas, como um modo de obter acesso a uma enorme variedade de tecnologias que nenhuma empresa consegue dominar sozinha e é necessária para desenvolver e lançar novos medicamentos a curto prazo (Wortzel, 1971; Taggart, 1993; Bierly III \& Chakrabarti, 1996; Kuemmerle, 1997; McCutchen Jr. \& Swamidass, 1998; Prabhun, 1999; Tapon \& Thong, 1999).

$\mathrm{Na}$ realidade, a sofisticação tecnológica é derivada do nível de capacidade tecnológica da empresa. Assim, o principal objetivo das empresas ao adquirirem conhecimento tecnológico externo é melhorar sua capacidade tecnológica (Leonard-Barton, 1995). Se esse objetivo for alcançado, significa que a tecnologia transferida externamente foi bem-sucedida.

No entanto, estudos mostram que nem sempre a aquisição de tecnologias externas é bem-sucedida. A transferência de tecnologia é uma atividade complexa, cujo sucesso depende de uma série de fatores.

Observando os vários fatores de sucesso da transferência de tecnologia, duas questões se colocam: como e quais fatores influenciam no sucesso da transferência de tecnologia nas empresas da indústria farmacêutica? É, portanto, com essas indagações e visando contribuir para a discussão do tema sucesso da transferência de tecnologia que os objetivos deste artigo foram definidos. Este estudo tem por objetivo geral analisar os fatores e as relações que afetam o sucesso da transferência de tecnologia em empresas da indústria farmacêutica. Os objetivos específicos são: 1 . verificar qual a extensão dos fatores internos (capacidade de absorção e capacidades gerenciais) e os modos de transferência que influenciam a habilidade da empresa em desenvolver capacidade tecnológica por meio de tecnologia importada; e 2. verificar em que medida as diferenças nos níveis de desempenho alcançados nas empresas farmacêuticas podem ser atribuídas a sua habilidade em desenvolver capacidades tecnológicas por intermédio da tecnologia importada.

Considerando essas transformações no cenário do processo de produção de medicamentos, neste trabalho serão analisados os fatores e as relações que influenciam o sucesso da transferência de tecnologia em três empresas farmacêuticas no Canadá. Para alcançar esse objetivo construiu-se um modelo conceitual. A pretensão não é, absolutamente, construir um modelo conceitual definitivo para essa questão, mesmo porque serão considerados os principais fatores provenientes da literatura.

Visto que a indústria farmacêutica e o processo de produção de medicamentos não são familiares para os leitores, o artigo começa com um resumo dessas características. A terceira parte apresenta uma revisão da literatura sobre os fatores e o sucesso na transferência da tecnologia. A quarta parte apresenta o modelo conceitual, construído a partir da revisão da literatura. A quinta parte descreve a metodologia dos estudos de casos realizados em empresas farmacêuticas canadenses. A sexta parte exibe os resultados dos estudos de casos. A sétima parte apresenta a análise desses resultados. A oitava e última parte apresenta as conclusões e as recomendações para trabalhos futuros.

\section{Características da indústria farmacêutica}

Uma das características da indústria farmacêutica é sua estrutura oligopolizada diferenciada com base na ciência e dominada por empresas transnacionais (Tavares, 1991; Camara, 1993). Segundo Camara (1993) e Bermudez (1995), as empresas farmacêuticas exercem poder oligopolístico em diferentes classes terapêuticas (antitérmico, analgésico, antidepressivo, antidiabético, cardiotônicos etc.), em que cada medicamento atende a um conjunto limitado de indicações, resultando num baixo grau de substituição entre si. De acordo com os dados da publicação IMS Health (2002), as 
classes terapêuticas líderes mundiais no ano 2001 foram em ordem decrescente de vendas: 1 . antiúlcera, 2 . colesterol e triglicérides, 3 . antidepressivo, 4. antireumático, 5. analgésico, 6. antipsicótico e 7 . antidiabéticos orais.

$\mathrm{O}$ mercado farmacêutico mundial faturou, em 1999, US\$201,3 bilhões (Bermudez, 2002). Esse mercado de produtos para uso humano costuma ser classificado em medicamentos éticos e nãoéticos (Camara, 1993; Queiroz, 1993).

Os medicamentos não-éticos, chamados de OTC (Over-The-Counter), representam pequena parcela do mercado, as técnicas de produção são simples, o conhecimento científico tecnológico é bastante difundido e sua inovação se restringe às formas de preparação e apresentação (Queiroz, 1993).

Os medicamentos éticos incluem o conjunto de medicamentos vendidos exclusivamente com receita médica e podem ser subdivididos em medicamentos genéricos e patenteados. Os genéricos são os que tiveram sua patente expirada e que passaram a ser fabricados por outros produtores além do que detinha a patente original. Eles compõem parcela menor, porém não desprezível do mercado. Os medicamentos patenteados ou de marca são os mais recentes e tecnologicamente mais avançados. Segundo Queiroz (1993), isso exige elevados investimentos em atividades de pesquisa e desenvolvimento $(\mathrm{P} \& \mathrm{D})$ e pessoal altamente qualificado. O mercado mundial de medicamentos éticos cresceu 7\% nas vendas em dólares em 1998, alcançando o total de US\$ 302 bilhões (Strategis, 2002). Segundo dados da publicação do IMS Health (2002), em 1999, os Estados Unidos mantinham a posição de maior mercado, com vendas de US\$130,1 bilhões (41,3\%), seguido do Japão, com US\$ 53,5 bilhões (15,9\%), e da Alemanha, com US\$18,5 bilhões (5,5\%). O Brasil estava na $8^{\mathrm{a}}$ posição, com US\$ 6,2 bilhões $(1,8 \%)$, e o Canadá na $10^{\mathrm{a}}$, com vendas de US\$ 5,5 bilhões $(1,7 \%)$.

Segundo dados publicados pela Strategis (2002), o Canadá está cada vez mais atraindo significativos investimentos de empresas farma- cêuticas nacionais e estrangeiras em pesquisa e manufatura. Os principais investimentos foram feitos recentemente pela Astra Zeneca, Paster Merieux Connaught, Hoechst Marion Roussel, Glaxo Wellcome, Amgen e Apotex.

\subsection{O processo de produção de medicamento}

Pesquisadores geralmente identificam cinco estágios principais no processo de produção de medicamentos: a) descoberta do fármaco, b) testes pré-clínicos animais, c) testes clínicos humanos, d) produção e e) comercialização.

Segundo Pisano (1997), o estágio de descobrimento de novos fármacos tem por missão identificar moléculas que comprovem segurança e eficácia no tratamento de doenças. O estágio é altamente dependente de tentativa e erro, de 10.000 moléculas exploradas, somente 20 são julgadas promissoras para garantir a continuidade do próximo estágio, o desenvolvimento pré-clínico (testes em animais). Atualmente, a ênfase está em planejamentos racionais de fármacos, em que moléculas são desenvolvidas a partir de teorias sobre a origem e a evolução de doenças e o uso da biotecnologia (Tapon \& Thong, 1999). A revolução tecnológica trazida pelos avanços na biotecnologia, na engenharia genética e na informática permite a produção de cerca de 50.000 novos componentes moleculares por ano, por meio de análises combinatórias e testes realizados nos computadores, dispensando, em grande parte, as bancadas dos laboratórios (Tapon \& Thong, 1999).

De acordo com a literatura, o estágio de testes clínicos é o que utiliza mais recursos, com duração em média de seis anos. Esse estágio é dividido em três fases, nas quais são realizados testes em humanos com o objetivo de determinar a eficácia e segurança do fármaco em pacientes humanos. A cada fase que o fármaco é aprovado, deve ser elaborada extensa documentação, descrevendo detalhadamente os procedimentos realizados, tanto em nível de teste quanto de fabricação do produto ainda em escala semi-industrial. Assim, a cada fase aprovada, 
maior é a probabilidade de que o fármaco alcance o mercado. Paralelo a esse estágio, em que as doses são determinadas e as formas de apresentação são estabelecidas (comprimidos, cápsulas, xaropes etc.), ocorre o desenvolvimento do processo da escala laboratorial para fabricação do medicamento em escala comercial. Nessa fase ocorre a maior concentração de profissionais com diferentes competências (farmacêuticos, químicos, engenheiros etc.). Segundo Pisano (1997), o conceito de engenharia simultânea é totalmente válido no processo de produção de medicamentos. Assim, quanto mais cedo os estágios de testes clínicos e desenvolvimento do processo trabalharem em paralelo, menores serão os custos e o lead time.

Uma vez bem-sucedidos os testes clínicos e validado e documentado o processo de fabricação, a empresa envia toda a documentação para uma autoridade de regulamentação do país que está sendo desenvolvido o medicamento, a fim de obter permissão para vendê-lo.

Percebe-se que o processo de produção de um medicamento é complexo. Segundo Raimundo (2001), para desenvolver e comercializar um medicamento leva de 12 a 15 anos, a um custo de US\$ 500 milhões teoricamente. A Phrma (2000) estimou que em 1999 as empresas farmacêuticas dos Estados Unidos investiram cerca de $21 \%$ de suas vendas em pesquisa. Os investimentos em P\&D de medicamentos no Canadá têm crescido nos últimos anos. Em 1999 foram gastos US\$ 5,5 bilhões, aumento de 11\% em relação a 1996 (IMS Health, 2002). Quanto ao Brasil, os investimentos em P\&D, em 1999, foram de US\$ 66 milhões, aumento de 50\% em relação ao ano interior, e de $214 \%$ em relação a 1992, quando os investimentos chegaram a US\$ 21 milhões (Interfarma, 2002).

No cenário de alta competição entre as empresas farmacêuticas, seja em qualquer segmento de mercado, a identificação, a transferência e a absorção da tecnologia mais apropriada podem contribuir significativamente para o sucesso do processo de produção de um novo medicamento, assim como reduzir seu alto custo e seu longo prazo de desenvolvimento.

\section{Revisão da literatura: fatores e sucesso da transferência de tecnologia}

Transferência de tecnologia pode ser definida como um processo pelo qual o conhecimento tecnológico passa de uma fonte para um recebedor, vertical ou horizontalmente (Al-Ghailani \& Moor, 1995).

A transferência de tecnologia é reconhecida como um processo complexo que engloba a identificação da tecnologia a ser transferida, a seleção dos modos (joint ventures, cooperação de pesquisa, fusões, licenciamento etc.) e mecanismos de transferência (treinamento, seminários, software, informações técnicas quanto ao uso e à manutenção da tecnologia, intercâmbio de profissionais etc.) e a completa implementação e absorção da tecnologia (Teece, 1976; Wallender, 1979; Contractor \& SagafiNejag, 1981; Contractor, 1985; Westphal et al., 1985; Al-Ghailani, 1995; Autio \& Laamanen, 1995). Além disso, segundo Souza (1981), a transferência será real se o recipiente for capaz de absorver, adaptar e melhorar os conhecimentos tecnológicos adquiridos.

Um dos temas mais comuns abordados na literatura de transferência de tecnologia é o sucesso da tecnologia transferida, o qual pode ser medido de várias maneiras, por exemplo: o desempenho alcançado em nível econômico, desenvolvimento de produto, produção e mercadológico (Yin, 1992; Kumar et al., 1999; Shrader, 2001), nível de satisfação obtida com a tecnologia transferida (McCutchen Jr. \& Swamidass, 1998) e nível de domínio tecnológico alcançado (Baranson \& Roak, 1985; Westphal et al., 1985; Leonard-Barton, 1995; Kumar et al., 1999). Dentre as várias medidas, a que apresenta maior consenso entre os autores está o nível de domínio tecnológico obtido pela empresa recebedora com a aquisição de conhecimento tecnológico externo. Cada nível de domínio tecnológico alcançado envolve a promoção de capacidades tecnológicas.

Leonard-Barton (1995) identificou que, no caso das empresas multinacionais, a transfe- 
rência de tecnologia para suas filiais proporciona níveis específicos de domínio tecnológico, o qual permite o cultivo de quatro níveis de capacidades: 1. transferência da capacidade operacional da planta em pleno funcionamento, 2. transferência da capacidade de adaptar o produto e de fabricá-lo usando principalmente componentes locais, 3. transferência da capacidade de redesenhar um produto integralmente em co-desenvolvimento com a matriz e 4 . transferência da capacidade de projetar um produto integral e independentemente da matriz e ter o potencial para a transferência inversa. Baranson \& Roak (1985) distinguem três capacidades entre as situações de transferência: as operacionais, as duplicadoras e as inovadoras. Mais recentemente, Kumar et al. (1999) distinguiram, a partir de projetos de transferência, a construção de três capacidades: capacidade de investimento, capacidade operacional e capacidade de aprendizagem dinâmica.

Apesar de terminologias diferentes, pesquisadores são unânimes em afirmar que nenhuma dessas capacidades tecnológicas é obtida automaticamente, elas requerem contínuo processo de aprendizagem (Desai, 1984; Westphal et al., 1985; Baranson \& Roak, 1985; Mytelka, 1985; Al-Ali, 1995; Al-Ghailani \& Moor, 1995; Baba \& Hatashima, 1995; Leonard-Barton, 1995; Kumar et al., 1999). Nesse sentido, considerando o modelo de Kumar et al. (1999), as três capacidades (investimento, operacional e aprendizagem dinâmica) podem ser obtidas de um único projeto de transferência de tecnologia, desde que haja, entre o desenvolvimento de uma capacidade e outra, acumulação de aprendizagem dentro da empresa recebedora da tecnologia. Para diversos autores, o processo de aprendizagem contínuo é de responsabilidade gerencial (Cusumano \& Elenkov, 1994; Leonard-Barton, 1995; Bessant \& Rush, 1995; Levin, 1997; Mcclements \& Smallman, 1998; Khan, 1999; Chapman, 2001).

Estudos têm identificado alguns fatores como relevantes para o sucesso da tecnologia transferida pela empresa recebedora. A literatura classifica esses fatores em internos e externos (Wallender,
1979; Yin, 1992; Arora \& Gambardella, 1994; Leonard-Barton, 1995; Wong, 1995; Tsang, 1995; Wei, 1995; Al-Ghailani, 1995; Kurokawa, 1997; Kuemmerle, 1997; Levinson \& Asahi, 1997; Mangematin \& Nesta, 1999; Kumar et al., 1999). Os fatores externos são: caracte- rísticas de infraestrutura, competição no mercado, modos de transferência, fator cultural e papel do governo. E os fatores internos são: capacidade de absorção, experiência da empresa, disponibilidade de treinamento, características estruturais da empresa e capacidades gerenciais.

Essas literaturas também mostram que o sucesso da tecnologia transferida é determinado por uma combinação de fatores. No entanto, cada estudo estabelece a combinação de fatores a ser analisada. Por exemplo, Kumar et al. (1999) investigou em empresas de diversos setores de negócio na Indonésia a construção de capacidades tecnológicas a partir de tecnologia transferida. Nesse estudo os autores combinaram fatores externos: envolvimento do governo, infra-estrutura e modos de transferência, e fatores internos: investimento em $\mathrm{P} \& \mathrm{D}$, disponibilidade de treinamento, atividades de planejamento e controle e qualificação dos empregados. Os autores concluíram que a construção de capacidades tecnológicas nas empresas da Indonésia era afetada pelos seguintes fatores: atividades de planejamento e controle, disponibilidade de pessoal qualificado, programas de treinamento e um específico modo de transferência, o de licenciamento.

\section{Modelo conceitual}

O objeto de estudo neste trabalho é analisar os fatores e as relações que afetam o sucesso da tecnologia transferida em empresas da indústria farmacêutica.

Analisando os vários estudos encontrados na literatura de transferência de tecnologia, verificouse que estes analisam o sucesso da transferência a partir de uma combinação de fatores. Tendo em vista essa particularidade, optou-se por considerar neste estudo uma abordagem semelhante. Elaborou-se um modelo conceitual (Figura 1) que 
representa a estrutura teórica a fim de estudar o sucesso de projetos de transferência em empresas do setor farmacêutico. Ambas as variáveis e suas medidas utilizadas neste estudo são derivadas da literatura. As variáveis de análise consideradas no modelo são descritas a seguir.

\subsection{Medindo o sucesso da tecnologia transferida}

O sucesso da transferência de tecnologia neste estudo é definido pelo grau em que a empresa recebedora pode aumentar seu domínio tecnológico e/ou seu desempenho por meio da tecnologia importada.

\subsubsection{Capacidades tecnológicas}

Capacidade tecnológica “... se refere à habilidade de compreender, utilizar, adaptar e desenvolver tecnologia" (Wong, 1995).

Segundo Rosenberg et al. (1985) apud Alves Filho (1990), o desenvolvimento de capacidades tecnológicas é um processo de acumulação de conhecimento tecnológico. Nesta pesquisa, esse processo é avaliado quanto ao nível de absorção tecnológica nos seguintes aspectos: 1 . domínio de operar a tecnologia, 2. domínio de manter, 3. domínio de reparar, 4. domínio de modificar (know-how), 5. domínio de co-desenvolver um produto e/ou processo com o fornecedor da tecnologia e 6. domínio de inovar (LeonardBarton, 1995; Kumar et al., 1999).

\subsubsection{Desempenho alcançado com a tecnologia transferida}

Segundo Devine et al. (1985) apud Alves Filho (1990), desempenho significa o grau de sucesso em atingir objetivos estabelecidos, o que deveria ser medido pelos indicadores de desempenho.

Melhoramento tecnológico é um dos fatores determinantes no aumento da produtividade, da qualidade e da competitividade do mercado, porque permite uso mais eficiente da mão-deobra, do capital e dos materiais. Neste estudo, o desempenho alcançado foi indicado pelas empresas recebedoras em função do objetivo de ter empregado a tecnologia importada.

\subsection{Medindo os fatores de sucesso}

\subsubsection{Capacidade de absorção}

Cohen \& Levinthal (1990) conceituam capacidade de absorção como a habilidade de a

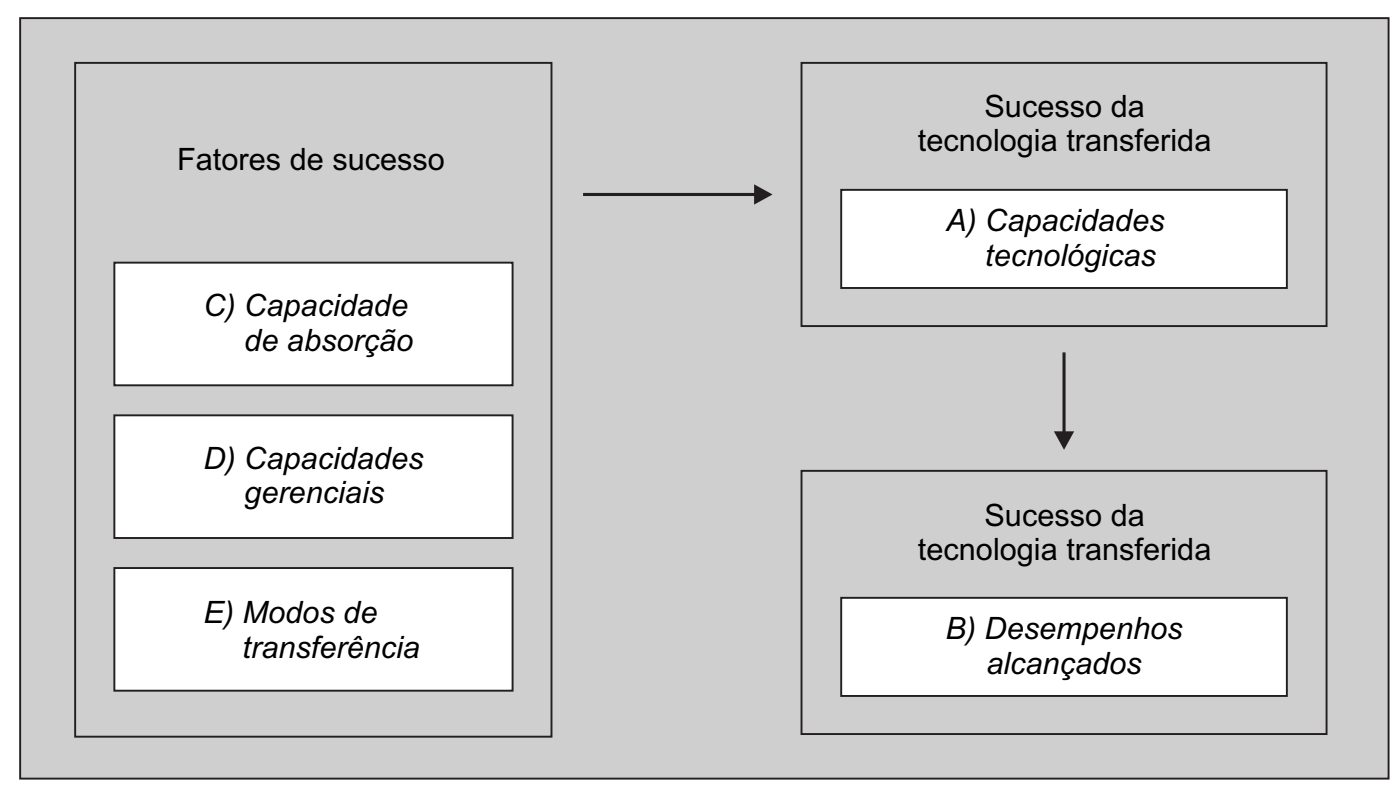

Figura 1 - Modelo conceitual. 
empresa reconhecer o valor de um novo conhecimento, assimilá-lo e aplicá-lo para fins comerciais. Ainda segundo os autores, o investimento em P\&D aumenta o estoque de conhecimento da empresa. No entanto, para Mangematin \& Nesta (1999), a natureza das tarefas diariamente realizadas por empregados altamente qualificados aumenta o estoque de conhecimento interno da empresa. Além disso, eles facilitam o acesso de conhecimentos externos, por intermédio de seu network com outros pesquisadores e cientistas de outras empresas, institutos de pesquisa e universidades, e pela participação em conferências. $\mathrm{O}$ número de patentes de uma empresa, considerando os resultados das atividades de $\mathrm{P} \& \mathrm{D}$, indica um conjunto de competências científicas que aumenta o valor da base de conhecimento (Mangematin \& Nesta, 1999). Em resumo, a caracterização da capacidade de absorção de uma empresa deve integrar os meios e os resultados das atividades de P\&D.

Como citado por Cohen \& Levinthal (1990), alta capacidade de absorção é, entretanto, uma condição permissiva para assimilar todo tipo de conhecimento e criar conhecimento. Nessa mesma linha, Mangematin \& Nesta (1999) citam que baixo nível de absorção implica que a empresa não pode assimilar conhecimento sem implementar fases de aprendizagem (criar estrutura de $P \& D$, aumentar nível de qualificação dos empregados, aumentar network externo). Os autores também citam que as empresas com alto nível de capacidade de absorção possuem condição de assimilar e criar novos conhecimentos.

Diversos autores são unânimes em afirmar que o nível de capacidade de absorção da empresa recebedora determinará a real extensão da transferência (Arora \& Gambardella, 1994; Leonard-Barton, 1995; Wong, 1995; Wei, 1995; Levinson \& Asahi, 1997; Mangematin \& Nesta, 1999). Para verificar o nível de capacidade de absorção de uma empresa, a literatura utiliza os seguintes indicadores: investimento em $P \& D$, número de pesquisadores (mestres e doutores), número de patentes, existência de parcerias com institutos de pesquisa e ou universidades (Cohen \& Levinthal, 1990; Arora \& Gambardella, 1994;
Leonard-barton, 1995; Levinson \& Asahi, 1997), os quais foram considerados neste estudo.

\subsubsection{Capacidades gerenciais}

A capacidade gerencial compreende uma série de habilidades, conhecimentos e experiências que uma pessoa deve ter para desempenhar certas funções gerenciais eficazmente (Katz, 1986; Kanter, 1989; Mintzberg, 1994; Bartlett \& Ghoshal, 1997; Shuman \& Thamhain, 1996; Floyd \& Wooldridge, 1997).

Vários autores citam que sem habilidades de gerenciamento é impossível organizar a empresa para receber, utilizar e explorar os conhecimentos tecnológicos adquiridos externamente (Wallender, 1979; Kanter, 1989; Bessant \& Rush, 1995; Leonard-barton, 1995; Kuemmerle, 1997; Harris \& Kumra, 2000; Chapman, 2001). Para este estudo, a partir da literatura revisada que discute as habilidades inerentes ao gestor (gerente geral, gerente de projetos, gerente de P\&D etc.), buscaram-se aquelas que seriam mais importantes ao profissional responsável por um projeto de transferência tecnológica (Quadro 1). Ter competência nas habilidades, descritas no Quadro 1, proporciona ao gestor desempenhar importante papel para conseguir alcançar o sucesso na transferência de tecnologia nas empresas.

A literatura cita que atividades de planejamento e de controle eficazes empregadas pela empresa durante a implementação da tecnologia transferida garantem o bom processo da transferência. Essas variáveis são medidas quanto ao grau de detalhamento empregado em termos de aquisição da tecnologia, à preparação do ambiente para a implementação da tecnologia e ao grau do progresso tecnológico.

\subsubsection{Modos de transferência da tecnologia}

Os diferentes modos de transferência auxiliam a empresa recebedora da tecnologia a aprender e a desenvolver novos conhecimentos, habilidades e capacidades tecnológicas (Hamel, 1991; Lei, 1997). A quantidade de capacidade tecnológica transferida depende em particular do modelo escolhido (Contractor, 1985; Cusumano \& Elenkov, 1994; Al-Ghailani, 1995; Bierly III \& 
Chakrabarti, 1996; Kumar et al., 1999). Contudo, o modo de transferência pode, por sua vez, depender da complexidade da tecnologia e também da maturidade da indústria (Contractor, 1985; Cusumano \& Elenkov, 1994).

$\mathrm{Na}$ indústria farmacêutica os modos mais comuns são: joint ventures, cooperação de pesquisa, licenciamento, investimentos externos diretos (Taggart, 1993; Bierly III \& Chakrabarti, 1996; Kuemmerle, 1997; McCutchen Jr. \& Swamidass, 1998).

\section{Metodologia}

O presente trabalho teve por foco as empresas farmacêuticas canadenses que realizaram projetos de transferência tecnológica recentemente. Essas empresas estão voltadas para o desenvolvimento de novos medicamentos para uso humano. O processo de pesquisa foi realizado por meio de estudos de casos em três empresas farmacêuticas conceituadas em seu mercado de atuação, tanto no segmento quanto na classe terapêutica.

Os dados quali e quantitativos foram obtidos por intermédio do preenchimento de um questionário estruturado. Os participantes (um por empresa) responderam às questões com base em um único projeto de transferência, pelo qual foram responsáveis e, portanto, estiveram diretamente envolvidos, desde seu início até sua finalização. Para este fim, elaborou-se um questionário composto por quatro partes principais, com questões descritivas e de múltipla escolha. A primeira parte compreendeu as informações gerais da empresa (origem do capital, segmento de mercado, classe terapêutica e estágio tecnológico da produção de medicamentos).

\section{Quadro 1 - Habilidades gerenciais.}

\begin{tabular}{|c|c|}
\hline $\begin{array}{l}\text { Habilidades } \\
\text { gerenciais }\end{array}$ & Características \\
\hline Liderança & $\begin{array}{l}\text { Capacidade de orientar, de motivar, de influenciar e de inspirar confiança a sua equipe } \\
\text { de subordinados para a realização das atividades pertinentes ao projeto de transferência. }\end{array}$ \\
\hline Comunicação & $\begin{array}{l}\text { Capacidade de comunicar com clareza a equipe sobre os benefícios do projeto de } \\
\text { transferência para alcançar os objetivos da empresa e clareza na transmissão de } \\
\text { instruções e solicitações para a equipe realizar o projeto. }\end{array}$ \\
\hline Negociador & $\begin{array}{l}\text { Capacidade de negociar com as várias entidades que participam do projeto de } \\
\text { transferência (fornecedor da tecnologia, equipe e o restante da organização). }\end{array}$ \\
\hline Técnica & $\begin{array}{l}\text { Capacidade de entender as tendências tecnológicas e o ambiente de negócios da } \\
\text { empresa e ter credibilidade técnica com os cientistas e engenheiros da empresa. }\end{array}$ \\
\hline Empreendedor & $\begin{array}{l}\text { Capacidade de gerar um ambiente de desafio, de encorajar a experimentação e a } \\
\text { inovação, de estimular a equipe a usar novas técnicas para solucionar problemas e de } \\
\text { adaptar a equipe às mudanças exigidas com o projeto de transferência. }\end{array}$ \\
\hline Coordenador & $\begin{array}{l}\text { Capacidade de formar e gerenciar a equipe de trabalho (resolver os conflitos da equipe } \\
\text { no âmbito do projeto, mobilizar os esforços da equipe), de alocar os recursos } \\
\text { necessários às várias etapas do projeto e de delegar tarefas e parte das decisões sobre os } \\
\text { aspectos técnicos do projeto. }\end{array}$ \\
\hline Instrutor (coach) & Capacidade de fomentar o aprendizado ocorrido com o projeto de transferência. \\
\hline
\end{tabular}


A segunda parte compreendeu informações para avaliar a capacidade de absorção da empresa. A terceira compreendeu dados sobre um projeto específico de transferência (qual o modo de transferência utilizado, em qual estágio da produção do medicamento a tecnologia foi aplicada, o valor do investimento, a idade da tecnologia transferida e qual o objetivo com a aquisição da tecnologia). A quarta compreendeu informações sobre as características do entrevistado e sua opinião quanto às questões relacionadas às capacidades gerenciais, e a quinta e última parte, informações sobre o domínio tecnológico (capacidade tecnológica) e o desempenho alcançado com a tecnologia transferida. A descrição das variáveis, a capacidade de absorção, capacidade gerencial, a capacidade tecnológica e o desempenho alcançado e suas respectivas medidas são apresentadas no Quadro 2.

\section{Estudos de casos}

\subsection{Características gerais das empresas}

\section{Empresa A}

Esta empresa é uma companhia farmacêutica multinacional de capital inglês, cujos objetivos principais são manter e fortalecer o sucesso da pesquisa, do desenvolvimento e da comercialização nas quatro classes terapêuticas prioritárias, em que a empresa é líder: respiratória, cardiovascular, gastrointestinal e analgésico.

A empresa atua exclusivamente no segmento de medicamentos de prescrição de marca e nos estágios do desenvolvimento à produção de medicamentos.

\section{Empresa B}

Esta empresa é de capital canadense e seu objetivo principal é ser líder mundial como fornecedora de sistemas de controle de liberação para melhorar a administração oral de medicamentos na forma sólida. Sua atuação é exclusiva no segmento de medicamentos prescritos de marca e nos estágios do descobrimento de novos fármacos até produção. Desenvolve e comer- cializa produtos para as diversas classes terapêuticas, porém, as principais são: analgésico, antigripal e antiinflamátorio.

\section{Empresa C}

Esta também é uma empresa multinacional de capital americano. Seu objetivo é ser conhecida pelo desenvolvimento de seus medicamentos contra dor, prescritos a pacientes com câncer em estágio avançado.

A empresa atua tanto no segmento de medicamentos de prescrição de marca como no segmento de OTC, este último em menor proporção. Realiza todos os estágios tecnológicos, do desenvolvimento até a comercialização de medicamentos. Os medicamentos são desenvolvidos e comercializados principalmente para as classes: analgésico, antibiótico e antiácido.

\subsection{Fatores de sucesso da tecnologia transferida}

\subsubsection{Fatores de sucesso}

\subsubsection{Características dos projetos e os modos de transferência}

\section{Empresa A}

Esta empresa realizou o projeto de transferência em 1998 e o fornecedor da tecnologia não se tratava da matriz transferindo tecnologia para a filial.

A tecnologia adquirida foi desenvolvida a menos de dois anos e o modo de transferência escolhido foi a compra de equipamentos com sua implementação na planta pela empresa fornecedora, cujo investimento foi de US\$ 150 mil. As reuniões, o suporte técnico e os treinamentos por parte do fornecedor duraram seis meses após a assinatura do contrato. A nova tecnologia foi adquirida para ser aplicada no estágio de descobrimento de fármacos. Segundo a empresa, seus objetivos com a aquisição dessa tecnologia eram reduzir custos de P\&D e aumentar a velocidade das análises, permitindo empregar o método de planejamento racional de fármacos. 


\section{Quadro 2 - Descrição das variáveis e suas medidas.}

\begin{tabular}{|c|c|}
\hline Variável & Descrição da variável e medidas \\
\hline $\begin{array}{l}\text { Capacidade de } \\
\text { absorção }\end{array}$ & $\begin{array}{l}\text { Investimento em P\&D como uma \% do total das vendas. } \\
\text { Mestres e doutores como uma proporção do total de empregados. } \\
\text { Número de parcerias com institutos de pesquisa e universidades. } \\
\text { Número de patentes por ano. }\end{array}$ \\
\hline $\begin{array}{l}\text { Capacidade } \\
\text { gerencial }\end{array}$ & $\begin{array}{l}\text { Nível de competência no gerenciamento do projeto de transferência de tecnologia segundo as } \\
\text { habilidades: } \\
\text { Negociador: eficiência na negociação com o fornecedor e com o grupo. } \\
\text { Empreendedor: encorajou a inovação e a experimentação, encorajou o grupo a usar novas } \\
\text { técnicas de solucionar problemas, criou ambiente de desafio e ajudou os empregados a se } \\
\text { adaptarem ao ambiente de risco e incerteza. } \\
\text { Liderança: auxiliou o grupo a tomar decisões, auxiliou na solução de problemas, mobilizou } \\
\text { esforços do grupo, motivou o grupo a continuar durante as dificuldades, obteve suporte e } \\
\text { compromisso dos superiores e teve sensibilidade cross-cultural. } \\
\text { Coordenador: construiu grupos multidisciplinares, gerenciou conflitos, delegou tarefas, delegou } \\
\text { decisões sobre os aspectos técnicos do projeto, gerenciou cooperação entre as várias funções } \\
\text { envolvidas no projeto e organizou recursos humanos, materiais e serviços. } \\
\text { Instrutor (coach): analisou o progresso de cada fase do projeto, fomentou o aprendizado e o } \\
\text { desenvolvimento dos indivíduos por meio de treinamento, fomentou o aprendizado ocorrido } \\
\text { com o projeto entre os membros do grupo e fomentou o aprendizado ocorrido com o projeto do } \\
\text { grupo para a empresa. } \\
\text { Comunicação: explicou com clareza aos empregados os benefícios do projeto em alcançar os } \\
\text { objetivos da empresa, comunicou (escrita e oral) as instruções e solicitações para o grupo } \\
\text { realizar o projeto e gerenciou problemas de comunicação entre grupo de P\&D, grupo de } \\
\text { manufatura, grupo de suporte técnico e o fornecedor da tecnologia. } \\
\text { Técnica: tem credibilidade técnica com cientistas/engenheiros e farmacêuticos, entende as } \\
\text { tecnologias e as tendências e participa da busca para integrar soluções e inovações tecnológicas. } \\
\text { Cada item foi medido em uma escala Linkert, obedecendo ao intervalo de } 1 \text { (não totalmente } \\
\text { competente) a } 5 \text { (extremamente competente), e a média da avaliação foi usada como a variável } \\
\text { medida. }\end{array}$ \\
\hline
\end{tabular}

Capacidade Nível de detalhamento no gerenciamento do projeto de transferência de tecnologia segundo as gerencial

Capacidade tecnológica

Desempenho alcançado atividades de:

Planejamento: preparou plano de aquisição da tecnologia, verificou compatibilidade da nova tecnologia com outras existentes na empresa, providenciou programa de conscientização de mudança tecnológica, preparou os empregados para implementar a tecnologia e estabeleceu medidas para avaliar o projeto.

Controle: determinou os modos de atuar sobre os desvios do desempenho planejado, determinou as causas dos desvios e realizou ações corretivas.

Cada item foi medido em uma escala Linkert, obedecendo ao intervalo de 1 (muito incompleto) a 5 (muito detalhado) e a média da avaliação foi usada como a variável medida.

Domínio da tecnologia para operar, manter, reparar, modificar, co-desenvolver um produto com o fornecedor, co-desenvolver um processo com o fornecedor e inovar a tecnologia. Cada item foi medido em uma escala Linkert, obedecendo ao intervalo de 1 (muito baixo) a 5 (muito alto), e a média da avaliação foi usada como a variável medida.

Desempenho medido em função dos principais objetivos indicados pelos próprios entrevistados para terem investido no projeto de transferência. $\mathrm{O}$ desempenho alcançado foi medido obedecendo à escala: $100 \%-90 \% ; 80 \%-70 \% ; 60 \%-50 \% ; 40 \%-30 \% ; 20 \%-10 \% ; 5 \%-0 \%$. 


\section{Empresa B}

Esta empresa, assim como a empresa A, realizou o projeto em 1998 e a tecnologia adquirida também tinha sido desenvolvida a menos de dois anos. O modo de transferência utilizado foi o licenciamento, em co-desenvolvimento com a empresa fornecedora de um novo método de pesquisa a ser aplicado no estágio de descobrimento de fármacos, sob o investimento de US\$ 130 mil. Assim como a empresa A, após a assinatura do contrato, as reuniões, o suporte técnico e os treinamentos seguiram por 18 meses. Os objetivos da empresa eram aumentar a eficiência e a segurança dos produtos, reduzir os custos de P\&D, aumentar a capacidade de mudar o volume de produção do fármaco e reduzir o lead time do desenvolvimento do processo de fabricação piloto.

\section{Empresa C}

A empresa $\mathrm{C}$ utilizou o modo de transferência licenciamento, aplicado no estágio de testes clínicos. Os objetivos eram reduzir o lead time do desenvolvimento do processo de fabricação piloto e do processo em escala industrial, aumentar o número de produtos desenvolvidos e lançados (diferentes formas de apresentação do medicamento), reduzir o timeto-market, melhorar a posição de mercado, aumentar a capacidade de mudar o volume de produção e reduzir os custos de $\mathrm{P} \& \mathrm{D}$. $\mathrm{O}$ valor do investimento do projeto foi de US\$ 2 mil e o fornecedor da tecnologia não se tratava da matriz transferindo tecnologia para a filial. A empresa realizou o projeto em 1995 e a tecnologia adquirida foi desenvolvida em 1991. Assim como as empresas A e B, após a assinatura do contrato, as reuniões, o suporte técnico e os treinamentos seguiram por seis meses.

Apesar de as empresas B e C utilizarem o licenciamento como modo de transferência, os valores dos investimentos são muito diferentes. Em relação a essa questão, Fitzgerald (1992) ressalta que, no caso de licenciamento, o tempo de vida da patente do fármaco é crucial. A vida da patente de um fármaco tem início com seu descobrimento.
Assim, quanto mais tempo ele demora em alcançar significativa penetração no mercado (fase de descobrimento - empresa B), menos tempo estará disponível para geração de lucros e menor será o valor da licença. E, ainda, segundo o autor, quanto menor for o risco de o fármaco não ser lançado no mercado (fase de testes clínicos - empresa C), maior será o valor da licença. Assim, nesse contexto, é possível explicar por que o valor do investimento da empresa C é maior que o da empresa B.

\subsubsection{Capacidade de absorção}

Segundo os indicadores utilizados para medir o nível de capacidade de absorção das empresas (Tabela 1), há indícios de que as três empresas são detentoras da habilidade para assimilar e aplicar os novos conhecimentos adquiridos com a tecnologia importada. Ainda segundo a literatura, quanto maior for o nível de capacidade de absorção, maior será a habilidade da empresa para criar novos conhecimentos.

\subsubsection{O perfil e as capacidades gerenciais dos entrevistados}

\section{Perfil dos entrevistados}

A Tabela 2 apresenta o perfil dos entrevistados, mostrando que os das empresas $\mathrm{B}$ e C possuem alta qualificação técnica (doutores em farmácia) e são executivos com alta experiência na posição que ocupam, chefe científico e diretor de $\mathrm{P} \& \mathrm{D}$, respectivamente. Dos três entrevistados, o da empresa $\mathrm{C}$ é o que possui menos experiência com projetos de transferência.

\section{Capacidades gerenciais}

Quanto aos dados apresentados nas Tabelas 3 e 4, referentes às capacidades gerenciais, esclarecemos que foi solicitado aos entrevistados que: 1 . auto-analisassem suas competências quanto às habilidades relacionadas ao gerenciamento desse projeto de transferência em particular e 2. autoanalisassem o grau de detalhamento realizado nas atividades de planejamento e no controle do referido projeto. 
Tabela 1 - Níveis de capacidade de absorção das empresas.

\begin{tabular}{|c|c|c|c|}
\hline Indicadores da capacidade de absorção & Emp A & Emp B & Emp C \\
\hline$\%$ de investimento $\mathrm{P} \& \mathrm{D} / \mathrm{vendas}$ (ano) & $13 \%$ & $11 \%$ & $7 \%$ \\
\hline$\%$ de mestre e doutores/total de empregados & $30,4 \%$ & $23,6 \%$ & $5,5 \%$ \\
\hline $\mathrm{n}^{\mathrm{o}}$ de parcerias com institutos de pesquisa e/ou universidade & 4 & 2 & 1 \\
\hline $\mathrm{n}^{\mathrm{o}}$ de patentes (ano) & 11 & 8 & 3 \\
\hline
\end{tabular}

A Tabela 3 apresenta o resultado da média do nível de competência nas habilidades relacionadas ao gerenciamento do projeto de transferência, conforme descritas no Quadro 2.

A Tabela 3 mostra que, de maneira geral, o nível de competência das habilidades encontrase coerente com o nível hierárquico dos entrevistados. Por exemplo, percebe-se que o entrevistado da empresa $\mathrm{B}$, chefe científico, atribuiu notas mais altas a suas habilidades de liderança e técnica, e nota mais baixa a sua habilidade de negociador. $\mathrm{O}$ entrevistado da empresa $\mathrm{C}$ avaliou-se como possuidor de alta competência nas habilidades de negociador, empreendedor, comunicação e técnica, e possuidor de baixa competência na habilidade de instrutor.

A Tabela 3 também possibilita analisar a atividade gerencial que consiste em resolver problemas. Quanto maior for o nível de competência nas habilidades que o gerente possui, maior capacidade de resolver problemas complexos ele terá. Assim, para a solução de um problema no projeto de transferência que contém características de ordem técnica, de comunicação, de conflitos e de motivação, somente um gerente com alta competência em várias habilidades será capaz de resolvê-los de maneira mais eficaz.

A Tabela 4 apresenta o resultado da média do grau de detalhamento que o entrevistado teve com as atividades de planejamento e controle relacionadas ao projeto de transferência, conforme descritas no Quadro 2. Quanto maior for o detalhamento das atividades de planejamento e controle, maior será a evidência de que os responsáveis pelos projetos preparam suas empresas para receber e implementar as novas tecnologias.

\subsubsection{Sucesso da transferência tecnológica}

\subsubsection{Capacidades tecnológicas}

A Tabela 5 apresenta a capacidade tecnológica analisada a partir do nível de domínio tecnológico alcançado com o projeto de transferência pelas empresas $\mathrm{A}, \mathrm{B}$ e $\mathrm{C}$.

Tabela 2 - Perfil do entrevistado.

\begin{tabular}{lccc}
\hline & Emp. A & Emp. B & Emp. C \\
\hline Posição do entrevistado & Gerente geral & Chefe científico & Diretor de P\&D \\
Experiência na atual posição & 5 anos & 10 anos & 12 anos \\
Qualificação básica & Administração & Farmacêutico & Farmacêutico \\
Especialização & Comércio exterior & Doutor em farmácia & Doutor em farmácia \\
$\begin{array}{l}\text { Experiência em projetos de } \\
\text { transferência }\end{array}$ & 3 & 3 & 1 \\
\hline
\end{tabular}


Tabela 3 - Nível de competência nas habilidades gerenciais.

\begin{tabular}{lccc}
\hline \multicolumn{1}{c}{ Habilidades gerenciais } & Emp. A & Emp. B & Emp. C \\
\hline Negociador & 4 & 2 & 4 \\
Empreendedor & 4 & 4 & 4 \\
Liderança & 4 & $5^{*}$ & 3 \\
Coordenador & 4 & 4 & 3 \\
Instrutor (coach) & 3 & 4 & 2 \\
Comunicação & 4 & 4 & 4 \\
Técnica & 3 & $5 *$ & 4 \\
\hline
\end{tabular}

*5 - extremamente competente; 1 - não totalmente competente.

O sucesso da capacidade tecnológica da empresa é determinado pelo nível de domínio tecnológico alcançado. As empresas A e B mostram que o conhecimento tecnológico transferido foi incorporado em alto nível até o co-desenvolvimento de um produto e o co-desenvolvimento de um processo com o fornecedor de tecnologia, respectivamente. A empresa $\mathrm{C}$ apresenta somente alto nível de domínio tecnológico alcançado até o domínio de manter a tecnologia transferida.

\subsubsection{Desempenhos alcançados}

Os desempenhos alcançados pelas empresas A, $\mathrm{B}$ e $\mathrm{C}$ em função dos objetivos de empregar a tecnologia importada são apresentados na Tabela 6 .

\section{Análise dos estudos de casos}

\subsection{Capacidade de absorção - capacidade tecnológica}

Iniciamos a análise das informações expostas na seção anterior verificando se há alguma relação entre as capacidades de absorção das empresas e suas habilidades em desenvolver capacidade tecnológica a partir da tecnologia importada.

As empresas A, B e C apresentam uma série de características comuns, como, por exemplo, atuam em todos os estágios tecnológicos da produção do medicamento e também no mesmo segmento de mercado (medicamentos de prescrição de marca).

Outra característica é que as três empresas participam do mercado da classe terapêutica, analgésico. Embora haja semelhanças, são distintas no que se refere ao nível de capacidade de absorção (Tabela 1). Enquanto as empresas A e B demonstram possuir uma política voltada para ampliar seus níveis de capacidade de absorção, por intermédio de altos investimentos, tanto de recursos financeiros como humano, com o intuito de não serem somente detentoras da habilidade de assimilar e aplicar conhecimentos adquiridos externamente, mas também da habilidade de criar novos conhecimentos, a empresa $\mathrm{C}$ apresenta comportamento menos voltado para a habilidade de criar novos conhecimentos.

Tabela 4 - Planejamento e controle.

\begin{tabular}{lcccc}
\hline & Atividade & Emp. A & Emp. B & Emp. C \\
\hline Planejamento & 4 & 4 & 3 \\
Controle & 4 & $5^{*}$ & 3 \\
\hline
\end{tabular}

*5 - muito detalhado; 1 - muito incompleto. 
Tabela 5 - Capacidade tecnológica.

\begin{tabular}{lccc}
\hline \multicolumn{1}{c}{ Capacidades tecnológicas } & Emp. A & Emp. B & Emp. C \\
\hline Domínio para operar a tecnologia & $5 *$ & 4 & 4 \\
Domínio para manter a tecnologia & 4 & 4 & 4 \\
Domínio de reparar a tecnologia & 4 & - & - \\
Domínio de modificar a tecnologia & 4 & 4 & 3 \\
Domínio de co-desenvolver um produto com o fornecedor da & 4 & 4 & 3 \\
tecnologia & 3 & 4 & 3 \\
Domínio de co-desenvolver um processo com o fornecedor da & & & \\
tecnologia & 2 & 3 & 3 \\
Domínio de inovar a tecnologia & & & \\
\hline
\end{tabular}

* 5 - muito alto; 1 - muito baixo.

- não respondida, os entrevistados explicaram que não se aplicava ao tipo de tecnologia transferida.

Essas características das empresas evidenciam, ao relacionarmos as informações das Tabelas $1 \mathrm{e}$ 5, que parte da diferença da habilidade de desenvolver capacidade tecnológica está associada à diferença no nível da capacidade de absorção. Para obter elevado domínio em co-desenvolver um produto e/ou processo com o fornecedor da tecnologia e inová-la, a empresa também precisa apresentar ou buscar ser detentora da habilidade para criar novos conhecimentos, como podemos identificar pelos resultados obtidos das empresas A e B.

Tabela 6 - Desempenhos alcançados.

\begin{tabular}{lccc}
\hline \multicolumn{1}{c}{ Desempenhos } & Emp. A & Emp. B & Emp. C \\
\hline Aumentar a velocidade de análise & $100 \%-90 \%$ & - & - \\
Reduzir custos de P\&D & $100 \%-90 \%$ & $60 \%-50 \%$ & $40 \%-30 \%$ \\
Aumentar a segurança e a eficiência dos produtos & - & $100 \%-90 \%$ & \\
Aumentar a capacidade de mudar o volume de produção & - & $100 \%-90 \%$ & $20 \%-10 \%$ \\
$\begin{array}{l}\text { Reduzir o lead-time do desenvolvimento do processo de } \\
\text { fabricação piloto }\end{array}$ & - & $80 \%-70 \%$ & $60 \%-50 \%$ \\
$\begin{array}{l}\text { Reduzir o lead time do desenvolvimento do processo de } \\
\text { fabricação em escala industrial }\end{array}$ & - & - & $40 \%-30 \%$ \\
Melhorar a posição de mercado & - & - & $80 \%-70 \%$ \\
Reduzir time-to-market & - & - & $60 \%-50 \%$ \\
Aumentar o número de produtos desenvolvidos & - & - & $40 \%-30 \%$ \\
Aumentar o número de produtos lançados & - & & $40 \%-30 \%$ \\
\hline
\end{tabular}




\subsection{Modos de transferência - capacidade tecnológica}

Analisando os projetos de transferência realizados pelas empresas, são encontradas algumas características que poderiam ter tido efeito sobre a relação entre o modo de transferência e a habilidade da empresa de cultivar a capacidade tecnológica. Podemos mencionar a assessoria oferecida pelo fornecedor e em qual fase do estágio da produção do medicamento a tecnologia importada foi aplicada. Vamos analisar as empresas B e C que realizaram o mesmo modo de transferência, o licenciamento. O contrato realizado pela empresa B compreendia a importação de uma tecnologia recente (menos de 2 anos) a ser aplicada no estágio de descobrimento de fármacos, com período de 18 meses de atividades de assessoria pelo fornecedor (treinamentos, reuniões e suporte técnico). Além disso, o contrato estabelecia uma parceria entre as empresas para desenvolvimento de novas metodologias. Esses fatores podem ter permitido uma comunicação mais intensiva e gerado confiança entre as empresas, facilitando o aprendizado da empresa B, o que talvez tenha contribuído para que a empresa $\mathrm{B}$ alcançasse altos níveis de domínio tecnológico, desde operar a tecnologia até co-desenvolver um processo com o fornecedor da tecnologia (Tabela 5).

A empresa $\mathrm{C}$ realizou a transferência de uma tecnologia também recente, desenvolvida a menos de quatro anos e aplicada no estágio de testes clínicos. Estágio considerado tão complexo quanto o estágio de descobrimento. Segundo a literatura, em cada uma das três fases do estágio de testes clínicos é necessário o uso de uma série de metodologias e testes de bioequivalência e biodisponibilidade que buscam verificar a eficácia e a segurança do fármaco. Esse procedimento requer longo período para ser realizado. No entanto, o período de assessoria estabelecido no contrato foi de seis meses. Além disso, o contrato não compreendia parceria entre as empresas, como o da empresa B. Esse contexto parece ter gerado menor aprendizado, o que teria contribuído para que a empresa $\mathrm{C}$ não alcançasse altos níveis no domínio de modificar a inovar a tecnologia.

As características da tecnologia transferida pela empresa A são muito semelhantes às da empresa $\mathrm{B}$, tecnologia adquirida desenvolvida a menos de dois anos e aplicada no estágio de descobrimento. A diferença está no modo de transferência, compra e instalação da tecnologia. Apesar de esse modo parecer mais simples que o de licenciamento, a utilização da nova tecnologia e sua interação com as tecnologias existentes requer, por parte do fornecedor, treinamentos e suporte técnico de médio prazo. Segundo o entrevistado, o período de seis meses estabelecido no contrato foi adequado, pois os treinamentos permitiram aos empregados não somente manusear muito bem a nova tecnologia como também consertar sozinhos, sem recorrer ao fornecedor quando ocorria alguma irregularidade com seu funcionamento. Esse fator também pode ter tido efeito positivo no processo de aprendizado da empresa A, contribuindo com sua habilidade de desenvolver capacidade tecnológica.

\subsection{Capacidade gerencial - capacidade tecnológica}

Os casos apresentam, ainda, indicações importantes a respeito da influência da capacidade gerencial na habilidade da empresa em desenvolver capacidade tecnológica por meio de tecnologia importada. Podemos identificar que a capacidade gerencial, em especial as habilidades do gestor, também deve ser analisada levando em consideração o modo de transferência empregado.

$\mathrm{O}$ contrato de licenciamento utilizado pelas empresas $\mathrm{B}$ e $\mathrm{C}$ compreendeu as atividades de descobrimento e testes clínicos do fármaco, respectivamente, e não atividades de marketing. Nesse caso, as habilidades técnicas e de coordenador do gestor do projeto são importantes, porque será necessário formar um grupo com especialistas de várias áreas que junto com o gestor do projeto participarão diretamente da identificação da potencial candidata à licença (empresa e fármaco). 
A habilidade de coordenador possibilita ao gestor gerenciar melhor os conflitos entre os grupos das duas empresas, além de criar um ambiente de cooperação entre as várias funções envolvidas no projeto. As habilidades de liderança do gestor, como a de criar um ambiente de respeito mútuo, auxiliar o grupo a tomar decisões e solucionar os diversos problemas e motivar o grupo a estar totalmente envolvido com o projeto são fundamentais para a eficácia do mesmo. Em projeto de licenciamento, as informações trocadas entre os grupos devem permitir que ambas as partes entendam todas as instruções e solicitações. Portanto, gerenciar a comunicação para que seja a mais clara possível também possibilita o bom andamento do projeto. Além disso, para que os verdadeiros frutos do licenciamento sejam colhidos pela empresa, o processo de aprendizado da tecnologia deve ocorrer. A literatura cita que o processo de aprendizagem é uma responsabilidade gerencial. Assim, se o gestor fomentar o aprendizado ocorrido com o projeto entre os membros do grupo e do grupo para a empresa, ele auxiliará no desenvolvimento de uma nova capacidade tecnológica. No caso da empresa $\mathrm{B}$, há evidência de que os níveis de competência do entrevistado nas habilidades de liderança, coordenador, instrutor, comunicação e técnica contribuíram com a habilidade da empresa em desenvolver capacidade tecnológica por meio de tecnologia importada. Quanto à empresa C, os níveis de competência do entrevistado nas habilidades de liderança, coordenação e instrutor podem ter contribuído para que a empresa não alcançasse altos níveis de domínio em modificar e até inovar a tecnologia.

O modo de transferência, compra e instalação da tecnologia requer do gestor capacidade de entender as tendências tecnológicas, encorajar a experimentação, comunicar aos funcionários sobre os benefícios da nova aquisição tecnológica para com os objetivos da empresa, saber negociar o "pacote tecnológico" e promover um ambiente que inspire confiança para realização das atividades pertinentes ao projeto de transferência. $\mathrm{O}$ nível de competência nas habilidades de negociador, empreendedor, de comunicação e coordenador do gestor da empresa $\mathrm{C}$ podem ter contribuído para a empresa desenvolver capacidades tecnológicas, porém não há fortes evidências a esse respeito.

A implementação da tecnologia transferida também envolve um detalhado sistema de planejamento e controle. Segundo informações dos entrevistados das empresas A e B, houve preocupação em realizar um plano bem detalhado de aquisição da tecnologia, em verificar detalhadamente a compatibilidade da nova tecnologia em relação às outras tecnologias existentes e também em providenciar detalhadamente um programa de conscientização de mudança tecnológica. Além disso, detalharam os modos de atuar sobre os desvios do desempenho planejado e determinaram suas causas. No caso da empresa $\mathrm{C}$, o entrevistado informou que essas atividades foram realizadas, porém não houve preocupação com detalhes. $\mathrm{O}$ menor detalhamento estabelecido pela empresa $\mathrm{C}$ pode estar relacionado à pouca experiência do gestor do projeto de transferência. $\mathrm{O}$ gestor da empresa $\mathrm{C}$ participou de um único projeto antes deste, enquanto os gestores das empresas A e B haviam participado de outros três projetos.

A literatura cita que atividades de planejamento e controle eficazes empregadas pela empresa durante a implementação da tecnologia transferida garantem um processo de transferência eficaz. Assim, as empresas A e B, ao realizarem planejamento e controle mais detalhados do projeto de transferência (Tabela 4), contribuíram com suas empresas para alcançarem níveis mais altos de domínio tecnológico (Tabela 5).

\subsection{Capacidade tecnológica - desempenho alcançado}

Há evidências, quando se analisam as Tabelas 5 e 6, de que o nível de desempenho alcançado em função do objetivo de empregar a tecnologia importada está associado ao nível de desenvolvimento das capacidades tecnológicas. 
Podemos verificar que as empresas A e B alcançaram, em média, altos níveis de domínio tecnológico e que esses níveis possibilitaram a elas atingir os níveis de desempenho desejados. Os níveis de domínio tecnológico da empresa C são, em média, menores que os das empresas $\mathrm{A}$ e B (Tabela 5). Segundo o entrevistado da empresa C, os objetivos de reduzir os custos de $\mathrm{P} \& \mathrm{D}$, o lead time do desenvolvimento do processo de fabricação piloto e o lead time do desenvolvimento do processo em escala industrial e de aumentar o número de produtos desenvolvidos e lançados tiveram o nível de desempenho abaixo do esperado. Assim, a empresa C, com médio domínio tecnológico, pode ter influenciado no nível de desempenho obtido.

\section{Conclusões e recomendações futuras}

No que diz respeito ao desenvolvimento de medicamentos mais eficazes e seguros, o grau de sofisticação tecnológica está em alta. Observa-se que as empresas estão buscando, por intermédio da aquisição de tecnologia externa, aumentar seu domínio de novos conhecimentos tecnológicos necessários para desenvolver os medicamentos.

Este estudo teve por objetivo geral analisar os fatores (capacidade de absorção, capacidade gerencial e modo de transferência) e as relações que afetam o sucesso da transferência da tecnologia.

Apesar de a amostra estudada ser pequena, somente três empresas farmacêuticas, há evidências de que parte da diferença da habilidade em desenvolver capacidade tecnológica está associada à diferença no nível da capacidade de absorção. Níveis mais altos de capacidade de absorção indicam que a empresa busca não somente ser detentora da habilidade de assimilar conhecimentos, mas também de criá-los. Portanto, maior será a habilidade dessa empresa em alcançar altos níveis para dominar a inovação da tecnologia e, assim, desenvolver novas capacidades tecnológicas. Este trabalho, entretanto, não pôde confirmar a relação direta entre modo de transferência e capacidade tecnológica, mas apenas indicar a influência de dois elementos, a atividade de assessoria por parte da empresa fornecedora da tecnologia e o estágio da produção de medicamento. Os casos mostraram que quanto maior for o grau da atividade de assessoria em estágios complexos da produção de medicamento maior será a habilidade de a empresa recebedora da tecnologia desenvolver capacidade tecnológica. A análise da capacidade gerencial indicou que, para o modo de transferência licenciamento, um nível maior de competência do gestor nas habilidades de coordenador, técnica, liderança, instrutor e comunicador pode ter influenciado o desenvolvimento das capacidades tecnológicas, enquanto no modo de transferência de compra de equipamentos e implementação na planta foram as habilidades de negociador, comunicação, coordenador e empreendedor. Os casos também indicaram que as diferenças nos níveis de desempenho alcançados pelas empresas podem ser atribuídas a suas habilidades de desenvolver capacidades tecnológicas por intermédio de tecnologia importada. No entanto, para elaboração de análise mais adequada da relação entre essas variáveis, mais empresas devem ser estudadas.

A realização de novos estudos de casos nas empresas farmacêuticas no Brasil permitirá identificar outras relações entre os fatores, estabelecer tipologias de estratégias de transferência de tecnologia e verificar o sucesso da tecnologia transferida. Futuramente, novos resultados serão publicados com o objetivo de contribuir para a discussão sobre essa temática. 
AL-ALI, S. Developing countries and technology transfer. International Journal Technology Management, v. 10, n. 7/8, p. 705-713, 1995.

AL-GHAILANI, H. H.; MOOR, W. C. Technology transfer to developing countries. International Journal Technology Management, v. 10, n. 7/8, p. 687-703, 1995.

ALVES FILHO, A. G. Estratégia tecnológica, desempenho e mudança: estudo de casos em empresas da indústria de calçados. 1991. 189 f. Tese (Doutorado) - Escola Politécnica, Universidade de São Paulo, São Paulo.

ARORA, A.; GAMBARDELLA, A. Evaluating technological information and utilizing it: scientific knowledge, technological capability and external linkages in biotechnology. Journal of Economic Behaviour and Organization, v. 24, n. 1, p. 91-114, 1994.

AUTIO, E.; LAAMANEN, T. Measurement and evaluation of technology transfer: review of technology transfer mechanisms and indicators. International Journal Technology Management, v. 10, n. 7/8, p. 643-664, 1995.

BABA, Y.; HATASHIMA, H. Capability transfer in the Pacific Rim nations: the case of Japanese electrical and eletronics firms. International Journal Technology Management, v. 10, n. 7/8, p. 732-746, 1995.

BARANSON, J.; ROAK, R. Trends in north: South transfer of high technology. In: ROSENBERG, N.; FIRSCHTAK, C. (Eds.). International technology transfer: concepts, measures, and comparisons. New York: Praeger, 1985.

BARTLETT, C. A.; GHOSHAL, S. The myth of the generic manager: new personal competencies for new management roles. California Management Review, v. 40, n. 1, p. 92-116, 1997.

BERMUDEZ, J. A. Z. Indústria farmacêutica, estado e sociedade. São Paulo: Hucitec, 1995.

BERMUDEZ, J. Medicamentos: acesso e perspectivas na virada do século. Disponível em: <www. abrasco.org.br/boletim/bol76/bol76edi.htm>. Acesso em: 2002.

BESSANT, J.; RUSH, H. Building bridges for innovation: the role of consultants in the technology transfer. Research Policy, v. 24, p. 97-114, 1995.
BIERLY III, P. E.; CHAKRABARTI, A. K. Technological learning, flexibility, and new product development in the pharmaceutical industry. IEEE Transactions on Engineering Management, v. 43, n. 4, p. 368-380, 1996.

CAMARA, M. R. G. Indústria farmacêutica: grupos estratégicos, tecnologia e regulamentação à experiência brasileira em debate. 1993. 267 f. Tese (Doutorado) - Faculdade de Economia, Administração e Contabilidade, Universidade de São Paulo, São Paulo.

CHAPMAN, J. A. The work of managers in new organizational contexts. The Journal of Management Development, v. 20, n. 1, p. 55-68, 2001.

COHEN, W. M.; LEVINTHAL, D. A. Absorptive capacity: a new perspective on learning and innovation. Administrative Science Quarterly, v. 35, p. 128-152, 1990.

CONTRACTOR, F. Licensing versus foreign direct investment in U.S corporate strategy: an analysis of aggregate U.S. data. In: ROSENBERG, N.; FIRSCHTAK, C. (Eds.). International Technology Transfer: concepts, measures, and comparisons. New York: Praeger, 1985.

CONTRACTOR, F.; SAGAFI-NEJAG, T. International technology transfer: major issues and policy responses. Journal of International Business Studies, p. 113-135, 1981.

CUSUMANO, M. A.; ELENKOV, D. Linking International technology transfer with strategy and management: a literature commentary. Research Policy, v. 23, p. 195-215, 1994.

DESAI, A. V. India's technological capability: an analysis of its achievements and limits. Research Policy, v. 13, p. 303-310, 1984.

FITZGERALD, J. D. Technology transfer issues in licensing pharmaceutical products. $R \& D$ Management, v. 22, n. 3, p. 199-208, 1992.

FLOYD, S.; WOOLDRIDGE, B. Middle management's strategic influence and organizational performance. Journal of Management Studies, v. 34, n. 3, p. 465-485, 1997.

HAMEL, G. Competition for competence and interpartner learning within international strategic alliances. Strategic Management Journal, v. 12, p. 83-104, Summer, 1991. 
HARRIS, H.; KUMRA, S. International manager development: cross-cultural training in highly diverse environments. Journal of Management Development, v. 19, n. 7, p. 602-614, 2000.

IMS HEALTH. Disponível em: <www.ims_global. com/insight/world_in_brief/review99/na.htm>.

INTERFARMA. Disponível em: <www.interfarma. org.br/panorama/phrma.htm>.

KANTER, R. M. The new managerial work. Harvard Business Review, p. 80-92, nov.-dec. 1989.

KATZ, R. L. As habilitações de um administrador eficiente. Nova Cultura, v. 1, p. 57-92, 1986.

KHAN, M. Dynamic techno-management capability of Indian computer firms in comparation with Korea. Technovation, v. 19, p. 243-259, 1999.

KUEMMERLE, W. Building effective R\&D capabilities abroad. Harvard Business Review, v. 75, n. 2, p. 61-70, 1997.

KUMAR, V. et al. Building Technological Capability through importing technology: the case of Indonesian manufacturing industry. Journal of Technology Transfer, v. 24, p. 81-96, 1999.

KUROKAWA, S. Make-or-buy decisions in R\&D: small technology based firms in the United States and Japan. IEEE Transactions on Engineering Management, v. 44, n. 2, p. 124-133, 1997.

LEI, D.T. Competence-building, technology fusion and competitive advantage: the key roles of organizational learning and strategic alliances. International Journal Technology Management, v. 14, n. 2/3/4, p. 208-237, 1997.

LEONARD-BARTON, D. Wellsprings of knowledge. Boston, MA: Harvard Business School Press, 1995.

LEVIN, M. Technology transfer is organizational development: an investigation into the relationship between technology transfer and organizational change. International Journal Technology Management, v. 14, n. 2/3/4, p. 297-308, 1997.

LEVINSON, N. S.; ASAHI, M. Cross-National alliances and international learning. IEEE Engineering Management Review, Fall, p. 32- 41, 1997.

MANGEMATIN, V.; NESTA, L. What kind of knowledge can a firm absorb? International Journal Technology Management, v. 18, n. 3/4, p. 149-172, 1999.

McCLEMENTS, R.; SMALLMAN, C. Managing in the new millennium: reflections on change, management and the need for learning. Management Decision, v. 36, n. 1, p. 3-8, 1998.
McCUTCHEN Jr., W. W.; SWAMIDASS, P. M. Explaining the differences in domestic and crossboundary strategies alliances in the pharmaceutical biotech industry. International Journal Technology Management, Special Issue on Management of Technology in Health Care, p. 490-506, 1998.

MINTZBERG, H. Rouding out manager's job. Sloan Management Review, Fall, p. 14-26, 1994.

MYTELKA, L. Stimulating effective technology transfer: the case of textiles in Africa. In: ROSENBERG, N.; FIRSCHTAK, C. (Eds.). International Technology Transfer: concepts, measures, and comparisons. New York: Praeger, 1985.

OMTA, S.W.F. et al. Managing industrial pharmaceutical R\&D. A comparative study of management control and innovative effectiveness in European and Anglo-American companies. $R \& D$ Management, $\mathrm{v}$. 24, n. 4, p. 303-315, 1994.

PhRMA. Disponível em: 〈www.phrma.org > Acesso em: 2000.

PRABHU, G. N. Managing research collaborations as a portfolio of contracts: risk reduction strategy by pharmaceutical firms. International Journal Technology Management, v. 18, n. 3/4, p. 207-231, 1999.

PISANO, G. P. The development factory: unlocking the potential of process innovation. Boston: Harvard Business School Press, 1997.

QUEIROZ, S. R. R. Estudo da competitividade da indústria Brasileira: competitividade da indústria de fármacos. Campinas: Unicamp, 1993.

RAIMUNDO, J. Seminário Abifarma - Acesso aos Medicamentos no Brasil. Disponível em: $<\mathrm{Http} / / / \mathrm{www}$. abifarma.com.br/seminario>. Acesso em: 18 set. 2001.

SHRADER, R. C. Collaboration and performance in foreign markets: the case of young high-technology manufacturing firms. Academy of Management Journal, v. 44, n. 1, p.45-60, 2001.

SHUMAN, J. C.; THAMHAIN, H. J. Developing technology managers. In: GAYNOR, G. H. (Ed.). Handbook of technology management. New York: McGraw-Hill, p. 21.1-21.15, 1996.

SOUZA, J. A. Dinamização da transferência vertical da tecnologia: diagnóstico e reposição de uma alternativa. Simpósio de pesquisa em administração de ciência e tecnologia, 6. São Paulo: USP, 1981.

STRATEGIS. Disponível em: <http://strategis.gc. ca/>. Acesso em: 2002. 
TAGGART, J. The world pharmaceutical industry. London: Routledge, 1993.

TAPON, F.; THONG, M. Research collaborations by multi-national research oriented pharmaceutical firms: 1988-1997. R\&D Management, v. 29, n. 3, p. 219-231, 1999.

TAVARES, A. C. Mercado farmacêutico: um panorama da década de 80. Revista de Administração Pública, v. 25, n. 2, p. 40-56, 1991.

TEECE, D. J. The multinational corporation and Resource cost of international technology transfer. Cambridge, Massachusetts: Ballinger Publishing Company, 1976.

TSANG, E. W. K. The implementation of technology transfer in Sino-foreign joint ventures. International Journal Technology Management, v. 10, n. 7/8, p. 757-766, 1995.

WALLENDER, H. V. Technology transfer and management in the developing countries: company cases and policy analyses in Brazil, Korea, Peru, and Tanzania. Cambridge: Ballinger Publishing Company, 1979.
WEI, L. International technology transfer and development of technological capabilities: a theoretical framework. Technology in Society, v. 17, n. 1, p. 103-120, 1995.

WESTPHAL, L. E. et al. Reflections on the Republic of Korea's acquisition of technological capability. In: ROSENBERG, N.; FIRSCHTAK, C. (Eds.). International technology transfer: concepts, measures, and comparisons. New York: Praeger, 1985.

WONG, J. K. Technology transfer in Thailand: descriptive validation of a technology transfer model. International Journal Technology Management, v. 10, n. 7/8, p. 788-796, 1995.

WORTZEL, L. H. Technology transfer in the pharmaceutical industry. New York: UNITAR Research Report, n. 14, 1971.

YIN, J. Z. Technological capabilities as determinants of the success of technology transfer projects. Technological Forecasting and Social Change, v. 42, p. 17-29, 1992.

\section{PROPOSAL OF A CONCEPTUAL MODEL TO ANALYSIS OF SUCCESS OF TECHNOLOGY TRANSFER PROJECT: STUDY IN PHARMACEUTICAL FIRMS}

\section{Abstract}

The acquisitions of external technological knowledge have been a usual practice for the pharmaceutical firms developed drugs more effective and safe. Aiming to collaborate for the more knowledge about the success of technology transfer in these firms had been reviewed the subject factors of success in the technology transfer, and analyzed the applicability of a conceptual model in three firms in Canada.

Key words: technology transfer, technological capability, managerial capability, pharmaceutical firms. 